\title{
Effet de l'infection trypanosomienne sur les performances au travail des taurins Ndama trypanotolérants en zone subhumide du Sénégal
}

\author{
M.T. Seck ${ }^{1 *}$ A. Fall ${ }^{2}$ A. Diaité ${ }^{2}$ \\ A. Diokou ${ }^{1}$ M. Dieng ${ }^{1}$
}

Mots-clés

Bovin N'Dama - Résistance aux maladies - Trypanosoma

congolense - Infection expérimentale Performance animale - Sénégal.

\begin{abstract}
Résumé
Cette étude a eu pour objectif de mesurer l'impact de l'infection trypanosomienne sur les performances au travail des taurins trypanotolérants en zone subhumide du Sénégal. Elle a été menée sur des bœufs Ndama dont le poids vif moyen a été de $288 \mathrm{~kg}$ et l'âge moyen de six ans. Les animaux ont été dressés individuellement (monoboeuf). Le dispositif expérimental a comporté deux phases de quatre semaines chacune. La première a consisté à faire tirer à des animaux sains une charge équivalente à 12 p. 100 de leur poids vif, pendant cinq heures par jour et cinq jours par semaine. Dans une seconde phase, après trois semaines de repos, les animaux ont été infectés artificiellement par voie intradermique avec une souche de Trypanosoma congolense (dose infectante : $10^{5}$ trypanosomes par millilitre) et ont été soumis aux mêmes exercices. L'infection trypanosomienne a eu un effet significatif sur la puissance développée $(p<0,001)$, la vitesse de travail $(p<0,001)$, la distance parcourue $(p<0,001)$, le volume du culot de centrifugation $(p<0,05)$ et la consommation d'Andropogon gayanus $(p<0,01)$. En revanche, elle n'a pas eu d'effet significatif sur le poids vif des animaux. Les résultats ont montré que les animaux sains ont été plus performants au travail que les animaux infectés par les trypanosomes. Des recommandations en matière de soins sanitaires et de prophylaxie trypanosomienne sont proposées pour améliorer les performances au travail des taurins Ndama trypanotolérants utilisés pour le trait : en fin de saison sèche, traiter les animaux contre la trypanosomose avec de I'acéturate de diminazène (Bérénil ${ }^{\circledR}$ ) à la dose de $7 \mathrm{mg} / \mathrm{kg}$ et, pendant I'hivernage, les traiter avec de l'isométamidium à la dose de 0,5 à $1 \mathrm{mg} / \mathrm{kg}$ de poids vif pour les protéger durant la période de la campagne agricole.
\end{abstract}

\section{INTRODUCTION}

En Casamance, région située au sud du Sénégal, l'introduction de la traction animale a été plus tardive que dans le reste du pays. Cependant, dans cette région infestée de glossines, la traction bovine avec la race taurine trypanotolérante Ndama a longtemps été la seule forme possible de mécanisation agricole (8). Bien que trypanotolérants, ces taurins deviennent parfois vulnérables, ce qui

\footnotetext{
1. Institut sénégalais de recherches agricoles, Centre de recherches zootechniques de Kolda, BP 53, Kolda, Sénégal

2. Institut sénégalais de recherches agricoles, Laboratoire national d'élevage et de recherches vétérinaires de Dakar-Hann, BP 2057, Dakar, Sénégal

* Auteur pour la correspondance

Tél./fax : +2219961152; tél. : +2215593036

E-mail : mtseck@hotmail.com
}

affecte leur performance au travail, car la trypanotolérance est relative : son rôle dans le système de défenses immunitaires de l'animal peut être très réduit, en particulier lors d'infections fréquentes.

L'effet de la trypanosomose sur les performances au travail reste peu étudié. S'il est connu que les animaux infectés sont moins performants, aucune étude comparative avec des animaux indemnes de trypanosomoses n'a été menée de manière précise. Des études épidémiologiques dans la zone subhumide du Sénégal ont montré une incidence mensuelle de la trypanosomose de 7 p. 100 pour les animaux utilisés pour la traction animale (10). L'objectif de cette étude a été d'évaluer l'impact de l'infection trypanosomienne sur le travail des animaux et de recommander des traitements pour ces animaux de trait. Ceci afin d'éviter non seulement la perte de travail observée dans cette étude, mais aussi la perte même d'animaux et le manque à gagner des utilisateurs de la traction bovine en zone d'enzootie trypanosomienne. 


\section{MATERIEL ET METHODES}

\section{Animaux}

L'étude a porté sur huit taurins mâles castrés Ndama trypanotolérants, d'un âge moyen de six ans et originaires des villages du département de Kolda (Région de Kolda). Leur poids vif moyen a été de $288 \pm 5,2 \mathrm{~kg}$. Ils ont été dressés pour travailler individuellement (monoboeuf) afin d'éviter, lors du test, les effets de confusion inévitable avec une paire de bovins.

L'expérimentation s'est déroulée pendant l'hivernage, de juillet à octobre. L'alimentation des animaux a été à base d'Andropogon gayanus comme aliment de lest, de graines de coton comme supplément, complétée par un complexe minéral et vitaminé. Andropogon qui était fauché au jour le jour et distribué ad libitum était planté depuis 1978 dans une parcelle de 30 ha (5). Après la fauche, qui se faisait en plusieurs endroits de la parcelle, aucune observation n'a été faite sur les repousses. Les graines de coton ont été distribuées à raison de $22 \mathrm{~g} / \mathrm{kg} / \mathrm{P}^{0,75}$ par animal et par jour. Avant le début des tests, une période d'adaptation de deux semaines a été observée pour habituer les animaux à la nouvelle ration alimentaire. Le matin, avant le début des travaux, les graines de coton ont été distribuées aux animaux. Au retour du travail, Andropogon a servi d'alimentation jusqu'au lendemain. Les quantités d'aliment distribuées et refusées ont été mesurées quotidiennement. Les animaux ont été abreuvés matin et soir.

Avant le début des tests, les animaux ont fait l'objet d'un déparasitage interne à base de tartrate de morantel (Exhelm ${ }^{\circledR} 750 \mathrm{mg}$ ) à la dose de $7,5 \mathrm{mg} / \mathrm{kg}$ de poids vif (PV), et d'un déparasitage externe avec du Bayticol ${ }^{\circledR}$ pour on. Ensuite, ils ont été traités à l'oxytétracycline (Terramycine Longue Action ${ }^{\circledR}$ ) à la dose de $20 \mathrm{mg} / \mathrm{kg} \mathrm{PV}$, avant d'être vaccinés contre la péripneumonie contagieuse bovine, la pasteurellose bovine et le charbon bactéridien. Après ces traitements, les animaux ont été mis au repos pendant deux semaines avant le début de l'expérimentation.

Les harnachements ont été des jouguets monobovins tels qu'ils sont décrits par Drawer (6) et Hopfen (11). Des modifications ont été apportées sur quelques pièces pour mieux les adapter au gabarit des animaux.

\section{Infection des animaux}

Les infections expérimentales ont été faites avec une souche de Trypanosoma congolense isolée originellement au ranch de MadinaDiassa (sud de Bamako, Mali) sur un taurin Ndama. Cinq des huit bovins ont subi, en seconde phase expérimentale, une infection artificielle par injection par voie intradermique (7) d'une dose unique de
$10^{5}$ trypanosomes par millilitre, répartie en quatre points $(0,25 \mathrm{ml}$ par point) : deux au niveau de chaque coté de l'encolure. Avant de procéder à l'infection artificielle, les animaux ont tous été traités à l'acéturate de diminazène (Bérénil ${ }^{\circledR}$ ) à la dose de $7 \mathrm{mg} / \mathrm{kg}$ PV (solution aqueuse à 7 p. 100) en respectant le délai d'élimination du produit.

\section{Dispositif expérimental \\ Type de travail}

Des traîneaux ont été confectionnés pour contenir la charge à tirer lors du travail. Le travail a consisté à tirer un traîneau chargé pendant cinq heures sur un parcours de quatre kilomètres (sol argilosableux) en faisant des allers et retours. Les traîneaux ont porté une masse ajustée pour produire une force de traction fixée à 12 p. 100 $\mathrm{PV}$ et maintenue constante toute la semaine. Cette force de traction a reflété les conditions d'utilisation des animaux dans les villages. Une force de travail de 15 p. 100 PV pourrait être appliquée pour une courte durée, mais cette expérimentation a duré quatre semaines pour chaque phase. L'arrêt du travail a été décidé lorsque l'animal n'a plus été apte à tirer le traîneau, en montrant des signes de fatigue par des modifications nettes de comportement.

\section{Traitements}

Les deux types de traitement appliqués aux animaux soumis au travail ont concerné les animaux sains indemnes de trypanosomes et les animaux infectés artificiellement par des trypanosomes.

Au cours de la première phase de quatre semaines (phase de noninfection : $\mathrm{P}_{1}$ ), tous les animaux ont été sains, indemnes de trypanosomoses. Afin de minimiser les risques d'infection naturelle, des pièges biconiques ont été posés tout au long du parcours pour capturer les glossines. Les huit animaux ont travaillé cinq jours par semaine et cinq heures par jour (de $8 \mathrm{~h}$ à 13 h 30, intercalé d'un repos de 30 min à $11 \mathrm{~h} 00$ ).

Après la $\mathrm{P}_{1}$, une phase de repos de trois semaines a été observée afin de dissiper les effets résiduels du travail accompli et de permettre aux animaux de compenser le poids perdu. Au cours de cette période, les animaux ont été soumis à un travail léger, un jour sur trois, pour éviter la nécessité d'un autre dressage à la reprise du travail pour la phase d'infection artificielle $\left(\mathrm{P}_{2}\right)$.

Cinq animaux ont été infectés artificiellement trois jours avant le démarrage de la $\mathrm{P}_{2}$. Le même dispositif expérimental que celui de la $\mathrm{P}_{1}$ a été appliqué pour la conduite des travaux.

\section{Mesures et observations}

Le tableau I illustre les mesures et les observations relevées pour les différents paramètres étudiés, durant les deux phases de travail.

\section{Tableau I}

Mesures et observations relevées en fonction des paramètres étudiés

\begin{tabular}{|c|c|c|c|}
\hline Paramètre & Sigle & Fréquence & Méthode \\
\hline Hématocrite & Vcc & Tous les jours & Lecture sur diagramme Mikro-Hämatokrit \\
\hline Parasitémie & Parasitémie & Tous les jours & $\begin{array}{l}\text { Murray et coll., 1977, Trans. R. Soc. trop. } \\
\text { Med. Hyg. ; Paris et coll., 1982, Acta trop. }\end{array}$ \\
\hline Consommation & Consommation & Tous les jours & Quantité offerte - Quantité refusée \\
\hline Vitesse & $\mathrm{V}$ & Tous les jours & $\mathrm{V}=\mathrm{L} / \mathrm{T}(\mathrm{T}=$ temps $)$ \\
\hline Distance parcourue & $\mathrm{L}$ & Tous les jours & Mesure distance parcourue $(\mathrm{km})$ \\
\hline Force de traction moyenne & $\mathrm{F}$ & Toutes les semaines & Dynamomètre \\
\hline Poids vif & PV & Toutes les semaines & Bascule électronique Barlo \\
\hline Travail de traction & W & Toutes les semaines & $\mathrm{W}=\mathrm{F} * \mathrm{~L}$ \\
\hline Puissance moyenne & $P$ & Toutes les semaines & $\mathrm{P}=\mathrm{F} * \mathrm{~L} / \mathrm{T}$ \\
\hline
\end{tabular}




\section{Analyses de laboratoire}

Les animaux utilisés au cours de la première phase expérimentale étaient «neufs » (ils n'avaient pas eu de contacts récents avec les trypanosomes), donc indemnes d'infection trypanosomienne à Trypanosoma congolense. Des prélèvements de sang ont été effectués sur ces animaux et ont fait l'objet d'analyses parasitologique et sérologique. L'analyse parasitologique a été faite après centrifugation du sang dans une centrifugeuse microhématocrite, la présence éventuelle de trypanosomes a été recherchée par l'examen du buffy coat avec un microscope réglé en contraste de phase (13). Les premiers résultats parasitologiques ont été confirmés par une deuxième analyse sérologique (test Elisa/antigène trypanosome) (9). Le témoin négatif a été constitué par des sérums provenant d'animaux neufs originaires du département de Dahra (zone sylvopastorale indemne de tsé-tsé et de trypanosomose à T. congolense). Le choix définitif des animaux de l'expérimentation a été basé sur la valeur de la densité optique de leur sérum. Tout animal dont le sérum a eu une densité optique égale ou supérieure à la moyenne de la densité optique du sérum des témoins plus deux écarts-types $(\mathrm{M}+2 \mathrm{sdv})$ a été jugé impropre à l'expérience à mener.

Des échantillons d'aliments distribués (Andropogon gayanus) ont été collectés tous les jours et la matière sèche (MS) a été déterminée. Ces différents échantillons ont été bien mélangés et des sous-échantillons ont été collectés pour déterminer la paroi cellulaire (PC), la lignocellulose (LC), l'azote $(\mathrm{N})$, les matières minérales $(\mathrm{MN})$, les autre matières cellulosiques et la matière organique (MO) (2).

\section{Analyses statistiques}

La puissance développée (P), le travail de traction (W), la vitesse de travail $(\mathrm{V})$, la force de traction $(\mathrm{F})$, la distance parcourue $(\mathrm{L})$, le volume du culot de centrifugation (Vcc), la consommation alimentaire et le poids corporel ont été analysés avec le logiciel SAS (17) en utilisant les modèles linéaires généralisés. Les facteurs inclus dans l'analyse de variance ont été le traitement (animal infecté ou non infecté), l'animal niché dans le traitement, la semaine et l'interaction entre ces termes.

\section{RESULTATS}

Les résultats présentés dans cette étude ont concerné, pour la $\mathrm{P}_{1}$, cinq animaux sains et, pour la $\mathrm{P}_{2}$, cinq animaux infectés artificiellement car, au cours de la $\mathrm{P}_{1}$, malgré les pièges biconiques posés tout le long du parcours, trois animaux sur les huit prévus initialement ont été infectés naturellement par Trypanosoma congolense. Leurs parasitémies ont été détectées aux $22^{\mathrm{e}}, 23^{\mathrm{e}}$ et $24^{\mathrm{e}}$ jours de travail. Lors des analyses statistiques, les données de ces trois animaux infectés naturellement n'ont été prises en compte ni dans la $\mathrm{P}_{1}$ ni dans la $\mathrm{P}_{2}$. Les résultats obtenus chez les cinq animaux sains lors de la $\mathrm{P}_{1}$ ont donc été comparés à ceux obtenus chez ces cinq mêmes animaux infectés artificiellement lors de la $\mathrm{P}_{2}$.

\section{Effet de l'infection trypanosomienne sur les niveaux de consommation et sur les performances pondérales}

L'ingestion d'aliments (Andropogon gayanus et graines de coton) est présentée dans le tableau II. L'ingestion moyenne d'Andropogon gayanus a été de $54,7 \mathrm{~g} \mathrm{MS} / \mathrm{kg} \mathrm{P}^{0,75}$ chez les animaux infectés artificiellement, alors qu'elle a été de $74,5 \mathrm{~g} \mathrm{MS} / \mathrm{kg} \mathrm{P}^{0,75}$ chez les animaux sains. La consommation moyenne de graines de coton a été respectivement chez les animaux sains et chez les animaux infectés de 14,8 g $\mathrm{MS} / \mathrm{kg} \mathrm{P}^{0,75}$ et de $16,3 \mathrm{~g} \mathrm{MS} / \mathrm{kg} \mathrm{P}^{0,75}$. L'infection trypanosomienne n'a pas eu d'effet significatif sur la consommation de l'aliment de supplémentation (graines de coton). Cependant, l'effet du traitement a été significatif $(\mathrm{p}<0,05)$ pour la consommation journalière d'Andropogon gayanus. Le tableau III montre que l'évolution du poids des animaux au cours des quatre semaines de travail n'a pas été affectée par le traitement appliqué. Les animaux sains ont maigri en moyenne de 7,95 kg par semaine et les animaux infectés de $6,76 \mathrm{~kg}$.

\section{Effet de l'infection trypanosomienne sur le volume du culot de centrifugation et la parasitémie}

La figure 1 montre l'évolution du Vcc des animaux. Au cours des périodes de travail, le Vcc des animaux sains est passé de 38 p. 100 à la première semaine à 36 p. 100 à la quatrième

\section{Tableau II}

Consommation d'Andropogon gayanus et de graines de coton chez les animaux sains et chez les animaux infectés

\begin{tabular}{|c|c|c|c|c|c|c|}
\hline \multirow[t]{2}{*}{ Variable } & \multirow[t]{2}{*}{ Semaine } & \multicolumn{2}{|c|}{ Animaux sains } & \multicolumn{2}{|c|}{ Animaux infectés } & \multirow[t]{2}{*}{$\mathbf{P}$} \\
\hline & & g MS/kg p po,75/j & g MS/100 kg PV/j & g MS/kg p p,75/j & g MS/100 kg PV/j & \\
\hline Andropogon & $\begin{array}{l}1^{\mathrm{e}} \\
2^{\mathrm{e}} \\
3^{\mathrm{e}} \\
4^{\mathrm{e}}\end{array}$ & $\begin{array}{l}74,5 \pm 4,5 \\
71,1 \pm 3,7 \\
75,7 \pm 3,7 \\
76,7 \pm 3,7\end{array}$ & $\begin{array}{l}1802 \pm 111,3 \\
1745 \pm 92,6 \\
1875 \pm 92,6 \\
1899 \pm 92,6\end{array}$ & $\begin{array}{l}59,7 \pm 3,7 \\
48,0 \pm 3,7 \\
56,7 \pm 4,6 \\
54,4 \pm 4,6\end{array}$ & $\begin{array}{l}1461 \pm 92,6 \\
1199 \pm 92,6 \\
1409 \pm 113,4 \\
1360 \pm 113,4\end{array}$ & $\begin{array}{l}* \\
* \\
* \\
*\end{array}$ \\
\hline Moyenne & & $74,5 \pm 2,0$ & $1830 \pm 48,8$ & $54,7 \pm 2,1$ & $1357 \pm 53,5$ & $*$ \\
\hline Graines de coton & $\begin{array}{l}1^{\mathrm{e}} \\
2^{\mathrm{e}} \\
3^{\mathrm{e}} \\
4^{\mathrm{e}}\end{array}$ & $\begin{array}{l}12,9 \pm 1,3 \\
14,2 \pm 1,1 \\
14,4 \pm 1,1 \\
17,5 \pm 1,1\end{array}$ & $\begin{array}{l}313 \pm 33,3 \\
348 \pm 27,7 \\
357 \pm 27,7 \\
434 \pm 27,7\end{array}$ & $\begin{array}{l}16,0 \pm 1,1 \\
15,3 \pm 1,1 \\
18,0 \pm 1,3 \\
16,5 \pm 1,3\end{array}$ & $\begin{array}{l}389 \pm 27,7 \\
381 \pm 27,7 \\
439 \pm 33,9 \\
412 \pm 33,9\end{array}$ & $\begin{array}{l}\text { ns } \\
\text { ns } \\
\text { ns } \\
\text { ns }\end{array}$ \\
\hline Moyenne & & $14,8 \pm 0,6$ & $363 \pm 14,6$ & $16,3 \pm 0,6$ & $406 \pm 16,0$ & ns \\
\hline $\begin{array}{l}\text { Consommation } \\
\text { totale de MS }\end{array}$ & & 89,3 & 2193 & 71,0 & 1763 & \\
\hline
\end{tabular}


Tableau III

Evolution du poids des animaux

\begin{tabular}{|lcccc} 
Variable & Semaine & $\begin{array}{c}\text { Animaux } \\
\text { sains }\end{array}$ & $\begin{array}{c}\text { Animaux } \\
\text { infectés }\end{array}$ & P \\
\hline Poids $(\mathrm{kg})$ & $1^{\mathrm{e}}$ & $288 \pm 5,2$ & $278 \pm 5,2$ & $\mathrm{~ns}$ \\
& $2^{\mathrm{e}}$ & $275 \pm 5,2$ & $256 \pm 5,2$ & $\mathrm{~ns}$ \\
& $3^{\mathrm{e}}$ & $265 \pm 5,2$ & $261 \pm 6,0$ & $\mathrm{~ns}$ \\
& $4^{\mathrm{e}}$ & $266 \pm 5,2$ & $256 \pm 6,0$ & $\mathrm{~ns}$ \\
Moyenne & & $274 \pm 2,6$ & $263 \pm 2,8$ & $\mathrm{~ns}$ \\
Variation & & & & \\
du poids & & -22 & -22 &
\end{tabular}

$\mathrm{ns}=$ non significatif

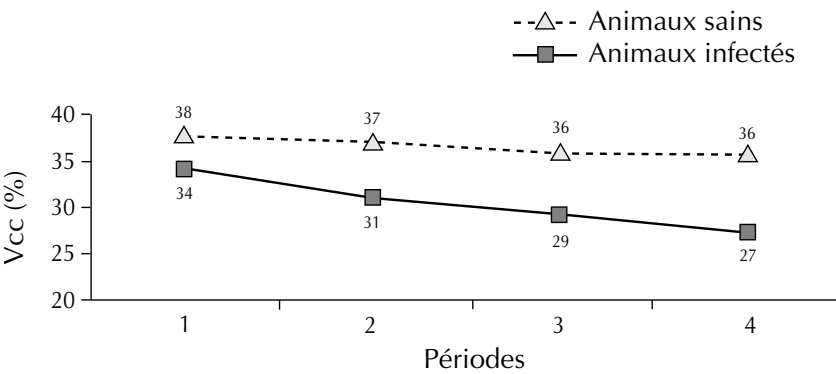

Figure 1 : évolution du Vcc au cours des quatre semaines de travail.

semaine, alors que celui des animaux infectés est passé de 34 à 27 p. 100. La moyenne du Vcc après quatre semaines de travail au cours de la première et deuxième phase a été respectivement de $36,6 \pm 0,4$ p. 100 et 30,4 $\pm 0,4$ p. 100 chez les animaux sains et chez les animaux infectés. Cet écart de valeurs entre les animaux $(6,2$ p. 100) montre un effet significatif $(p<0,05)$ de l'infection trypanosomienne sur le volume du culot de centrifugation. La figure 1 montre qu'il y a eu une diminution progressive du Vcc moins importante chez les animaux sains (0,96 p. 100 par semaine) que chez les animaux infectés (2,58 p. 100 par semaine). Au cours de la phase d'infection artificielle, la parasitémie $2+\left(10^{3}-10^{4}\right.$ trypanosomes/ml) est apparue en moyenne chez les animaux infectés au huitième jour après l'infection, donc après quatre jours de travail. Durant toute cette phase, la parasitémie a augmenté progressivement chez les animaux infectés sans pour autant dépasser la valeur $5+\left(>10^{5}\right.$ trypanosomes $\left./ \mathrm{ml}\right)$.

\section{Effet de l'infection trypanosomienne sur le travail fourni}

Le travail fourni a été en moyenne plus important chez les animaux sains $(6072 \pm 69,40 \mathrm{~kJ})$ que chez les animaux infectés (4 $263 \pm 81,23 \mathrm{~kJ}$ ) (figure 2). L'écart du travail fourni (1 $809 \mathrm{~kJ}$ ) entre les animaux sains et les animaux infectés montre un effet significatif $(p<0,001)$ de l'infection trypanosomienne sur le travail total. En moyenne, les animaux sains ont parcouru par jour une distance de 18,5 $\pm 0,21 \mathrm{~km}$ contre $13,6 \pm 0,25 \mathrm{~km}$ pour les animaux infectés (tableau IV). L'infection trypanosomienne a eu un effet significatif $(\mathrm{p}<0,001)$ sur la distance parcourue.

\section{Effet de l'infection trypanosomienne sur la puissance développée}

Les animaux infectés ont généré respectivement une puissance et une vitesse moyennes de $254,0 \pm 2,30 \mathrm{~W}$ et de $0,80 \pm 0,007 \mathrm{~m} / \mathrm{s}$

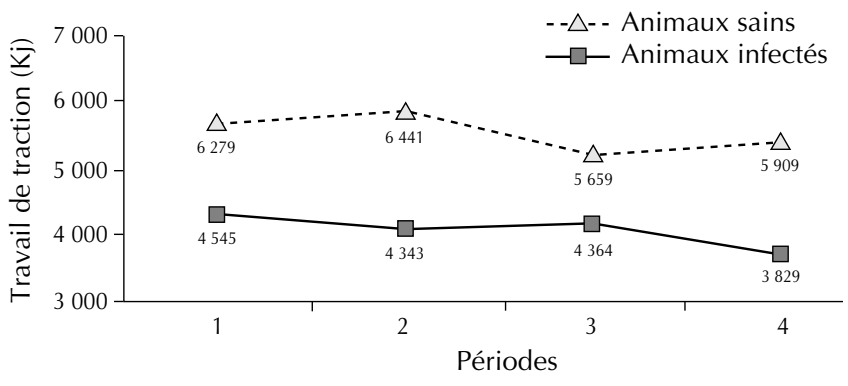

Figure 2 : variation du travail de traction au cours des quatre semaines.

\section{Tableau IV}

Effet de l'infection trypanosomienne sur la distance parcourue et la force de traction

\begin{tabular}{lcccc} 
Variable & Semaine & $\begin{array}{c}\text { Animaux } \\
\text { sains }\end{array}$ & $\begin{array}{c}\text { Animaux } \\
\text { infectés }\end{array}$ & $\mathbf{P}$ \\
\hline Distance & $1^{\mathrm{e}}$ & $18,1 \pm 0,43$ & $13,8 \pm 0,43$ & $* * *$ \\
par jour $(\mathrm{km})$ & $2^{\mathrm{e}}$ & $19,5 \pm 0,42$ & $14,0 \pm 0,42$ & $* * *$ \\
& $3^{\mathrm{e}}$ & $17,8 \pm 0,43$ & $14,0 \pm 0,52$ & $* * *$ \\
& $4^{\mathrm{e}}$ & $18,5 \pm 0,41$ & $12,5 \pm 0,52$ & $* * *$ \\
Moyenne & & $18,5 \pm 0,21$ & $13,6 \pm 0,25$ & $* * *$ \\
& & & & \\
Force & $1^{\mathrm{e}}$ & $346 \pm 1,54$ & $329 \pm 1,54$ & $\mathrm{~ns}$ \\
& $2^{\mathrm{e}}$ & $330 \pm 1,50$ & $307 \pm 1,50$ & $\mathrm{~ns}$ \\
& $3^{\mathrm{e}}$ & $319 \pm 1,54$ & $311 \pm 1,84$ & $\mathrm{~ns}$ \\
Moyenne & $4^{\mathrm{e}}$ & $319 \pm 146$ & $305 \pm 1,84$ & $\mathrm{~ns}$ \\
& & $327 \pm 0,75$ & $313 \pm 0,88$ & $\mathrm{~ns}$
\end{tabular}

*** $\mathrm{p}<0,001$

ns $=$ non significatif

(tableau $\mathrm{V}$ ). Les animaux sains se sont déplacés plus rapidement, avec une vitesse moyenne de $1,09 \pm 0,005 \mathrm{~m} / \mathrm{s}$, et ont généré une puissance plus élevée de l'ordre de 357,5 \pm 1,67 W. Il existe une différence significative $(\mathrm{p}<0,001)$ de la vitesse d'avancement ou de travail et de la puissance développée entre les animaux sains et les animaux infectés artificiellement.

\section{Comportement à l'effort des animaux sains et des animaux infectés}

Aussi bien chez les animaux sains que chez les animaux infectés, il a été observé au cours du travail des modifications nettes de leur comportement allant du ralentissement à l'augmentation de la fréquence des arrêts. Des positions couchées (décubitus latéral) ont été également observées chez les animaux infectés conduisant parfois à l'interruption du travail.

\section{DISCUSSION}

L'espèce de trypanosome choisie pour l'infection des animaux a été Trypanosoma congolense, espèce très pathogène et virulente. Dans la zone de Kolda, une étude épidémiologique a montré que 64,9 p. 100 des infections trypanosomiennes étaient dues à T. congolense (8). Cette espèce de trypanosome est connue pour avoir un impact économique énorme et pour provoquer souvent des infections chroniques au niveau du bétail (15). Pratiquement toutes les infections trypanosomiennes détectées à Kolda chez les glossines sont dues à $T$. congolense ou à $T$. vivax. En moyenne, 


\section{Tableau V}

Puissance et vitesse moyennes de travail des animaux sains et infectés au cours de quatre semaines de travail

\begin{tabular}{|c|c|c|c|c|c|c|}
\hline \multirow[t]{2}{*}{ Semaine } & \multicolumn{2}{|c|}{ Vitesse $(\mathrm{m} / \mathrm{s})$} & \multirow[t]{2}{*}{$\mathbf{P}$} & \multicolumn{2}{|c|}{ Puissance } & \multirow[t]{2}{*}{$\mathbf{P}$} \\
\hline & Animaux sains & $\begin{array}{l}\text { Animaux infectés } \\
\text { artificiellement }\end{array}$ & & Animaux sains & $\begin{array}{l}\text { Animaux infectés } \\
\text { artificiellement }\end{array}$ & \\
\hline $1^{e}$ & $1,08 \pm 0,01$ & $0,84 \pm 0,01$ & $* * *$ & $373 \pm 3,36$ & $281 \pm 3,95$ & $* * *$ \\
\hline $2^{e}$ & $1,14 \pm 0,01$ & $0,81 \pm 0,01$ & $* * *$ & $377 \pm 3,24$ & $250 \pm 3,84$ & $* * *$ \\
\hline $3^{e}$ & $1,08 \pm 0,01$ & $0,80 \pm 0,01$ & $* * *$ & $345 \pm 3,49$ & $252 \pm 4,69$ & $* * *$ \\
\hline $4^{e}$ & $1,05 \pm 0,01$ & $0,75 \pm 0,01$ & $* * *$ & $334 \pm 3,25$ & $233 \pm 4,93$ & $* * *$ \\
\hline Moyenne & $1,09 \pm 0,005$ & $0,80 \pm 0,007$ & $* * *$ & $357 \pm 1,67$ & $254 \pm 2,30$ & $* * *$ \\
\hline
\end{tabular}

2,9 p. 100 des mouches sont infectées chaque mois par T. vivax ou T. congolense. L'infection à T. congolense $(1,59 \mathrm{p}$. 100) est plus fréquente que celle à T. vivax (8).

Les résultats de cette étude ont montré, d'une part, une influence significative de l'infection trypanosomienne sur les niveaux de consommation d'Andropogon gayanus et, d'autre part, un effet non significatif sur l'évolution pondérale des animaux de trait. L'effet de l'infection sur le poids a été semblable aux résultats obtenus par l'Ilrad (12). La différence de consommation d'Andropogon gayanus (473 g MS/100 kg PV/j) entre les animaux sains et les animaux infectés peut être due, d'une part, à des facteurs intrinsèques aux animaux et, d'autre part, à des facteurs extrinsèques liés à l'aliment. Les facteurs liés à la qualité du fourrage consommé peuvent être écartés, car la composition chimique d'Andropogon gayanus fauché au jour le jour n'a pas présenté de différence significative entre les deux phases de l'expérience (tableau VI). Les valeurs des dernières fauches ont été relativement les mêmes que celles des premières fauches. En revanche, l'état de santé des animaux a probablement joué un rôle prépondérant dans la consommation du fourrage. Les animaux infectés perdent l'appétit, mais ne maigrissent pas plus vite que les animaux sains. Les animaux affaiblis par l'infection trypanosomienne ont tendance à moins consommer que les animaux sains. Ces derniers ont présenté les mêmes amplitudes ( $22 \mathrm{~kg}$ ) de diminution de poids que les animaux infectés. Pour ces animaux de trait, les pertes de poids seraient dues aux dépenses énergétiques liées à l'effort. Les animaux malades ont tendance à réduire leur effort de traction et leur puissance entrainnant une réduction de leur dépense énergétique liée au travail et par conséquent de leur perte de poids. Si l'expérience avait été prolongée de quelques semaines supplémentaires, une différentiation significative de l'évolution pondérale aurait probablement été observée dans le cas où les animaux ne se seraient pas bien alimentés.
L'hématocrite, qui révèle l'anémie, est un indicateur classique de la trypanosomose (4). Les résultats obtenus ont mis en évidence une influence significative $(\mathrm{p}<0,05)$ de l'infection trypanosomienne sur la valeur de l'hématocrite. Les animaux ont présenté au cours du travail des Vcc moyens élevés montrant leur capacité à résister à l'infection. Cela est probablement lié aux conditions alimentaires particulières auxquelles ils ont été soumis (ration alimentaire riche). Le Vcc des animaux infectés par les trypanosomes a diminué en moyenne de 2,58 p. 100 par semaine, comparé à celui des animaux sains ( 0,96 p. 100 par semaine). Ces résultats diffèrent de ceux obtenus par Agyemang et coll. (1) qui rapportent que le Vcc du bétail infecté avec des trypanosomes a diminué en moyenne de 4 p. 100 , comparé à celui du bétail non infecté $(0,5$ p. 100). Les écarts obtenus entre ces deux études relèvent de la différence d'activités soumises aux animaux. Le travail lui-même aurait un effet sur l'évolution du Vcc, car même les animaux sains ont montré une diminution de leur Vcc au cours du travail. Ces animaux, qui ont néanmoins un Vcc plus élevé, ont présenté les meilleures performances au travail. Les résultats de cette étude conduisent aux mêmes conclusions que celles rapportées par le CIPEA (3) : les animaux capables de maintenir leur hématocrite à des niveaux élevés s'avèrent toujours plus productifs que ceux présentant un hématocrite faible.

L'infection trypanosomienne a eu un effet significatif sur la distance parcourue. Les animaux sains ont parcouru une distance plus longue que les animaux infectés (18,5 km contre 13,6 km). Cette différence moyenne de distance parcourue $(4,9 \mathrm{~km} / \mathrm{j})$ entre ces deux catégories d'animaux se traduit par des diminutions de surfaces cultivées ou labourées pour le paysan qui utilise des animaux infectés par des trypanosomes. La baisse de la distance parcourue est due à une réduction de la vitesse de travail des animaux infectés. Ces animaux manifestent des signes de fatigue en modifiant leur comportement par un ralentissement de leur vitesse.

\section{Tableau VI}

Composition chimique d'Andropogon gayanus au cours de l'expérience

\begin{tabular}{lcrr} 
Composants analysés & Phase de non-infection & Phase de repos & Phase d'infection \\
\hline Matières sèches (g/kg produit brut) & & & 911 \\
Matières minérales (g/kg matière sèche) & 926 & 53 & 44 \\
Matières organiques (g/kg matière sèche) & 55 & 945 & 956 \\
Matières cellulosiques (g/kg matière sèche) & 388 & 395 & 404 \\
Matières protéiques (g/kg matière sèche) & 59 & 83 & 60 \\
Paroi cellulaire (g/kg matière sèche) & 779 & 476 & 490 \\
Lignocellulose (g/kg matière sèche) & 430 & 464 & 467
\end{tabular}


L'infection a eu un effet significatif sur le travail fourni. L'écart du travail (1 $809 \mathrm{~kJ})$ obtenu en comparant les performances de ces deux catégories d'animaux équivaut à 30 p. 100 et correspond à un manque à gagner si des animaux infectés sont attelés.

Les résultats de cette étude ont montré que les animaux sains ont avancé plus vite $(1,09 \mathrm{~m} / \mathrm{s})$ que les animaux infectés $(0,80 \mathrm{~m} / \mathrm{s})$. La puissance développée par les animaux sains $(357 \mathrm{~W})$ a été plus élevée que celle développée par les animaux infectés artificiellement $(254 \mathrm{~W})$. L'écart $(103 \mathrm{~W})$ entre ces deux types d'animaux est dû à leur différence de vitesse de déplacement. Il en résulte que l'infection trypanosomienne des taurins Ndama de trait entraîne une diminution de 29 p. 100 de la puissance développée.

L'évaluation de ces différents paramètres (vitesse, distance parcourue, travail fourni, puissance développée) entre les animaux infectés et les animaux sains permet d'apprécier leurs comportements et leurs adaptations physiologiques à l'effort fourni. L'animal infecté manifeste sa fatigue en modifiant son comportement : ralentissement, énervement. Cet effort de traction qui est un exercice musculaire entraîne une réduction de l'apport d'oxygène au niveau des muscles, se traduisant par une diminution de la puissance musculaire. Cette diminution de la puissance musculaire se traduit par une baisse de la puissance de travail liée à un ralentissement de la vitesse et, par conséquent, par une diminution du travail, pour une force de traction et une durée de travail fixes. Cette corrélation entre ces différents paramètres conduit à la conclusion évidente qu'un animal infecté par les trypanosomes travaille moins.

Avec ces baisses de performances, les paysans sont contraints de recourir à une main d'œuvre rémunérée, ce qui réduit leurs revenus. Cette contre-performance des animaux infectés peut probablement s'expliquer par un amoindrissement de leur état général dû à l'infection trypanosomienne, associée au travail qui est un facteur de stress pouvant diminuer la trypanotolérance, d'autant plus que celle-ci n'est pas absolue.

\section{CONCLUSION}

Les résultats obtenus dans cette étude ont montré que l'infection des taurins Ndama de trait par Trypanosoma congolense a eu un effet significatif sur la vitesse de travail ( $\mathrm{p}<0,001)$, la distance parcourue ( $\mathrm{p}<0,001)$, la puissance développée $(\mathrm{p}<0,001)$, le volume du culot de centrifugation $(\mathrm{p}<0,05)$ et la consommation d'Andropogon gayanus ( $<<0,01$ ). En revanche, l'infection n'a pas semblé affecter la perte de poids vif des animaux. Ces résultats ont aussi mis en évidence les meilleures performances au travail des animaux sains par rapport aux animaux infectés par les trypanosomes. L'infection trypanosomienne a entraîné une baisse des performances de travail des animaux de trait. Cette baisse de travail a comme conséquence au niveau des utilisateurs de la traction bovine une réduction des surfaces cultivées et une baisse des rendements, occasionnant une diminution considérable de leurs revenus pendant la campagne agricole. Cette baisse des performances des animaux due à l'infection trypanosomienne peut aboutir, avec les modifications nettes de comportement liées à l'effort fourni, à la perte des animaux de trait. Dans le contexte de zone subhumide où l'enherbement nécessite un labour préalable au semis, il est capital pour le paysan de disposer d'animaux rapides et puissants. Il apparaît nécessaire de formuler des recommandations en matière de protection sanitaire contre l'infection trypanosomienne. Un mois avant le début de l'hivernage, les animaux de trait doivent être traités contre la trypanosomose. L'acéturate de diminazène (Bérénil ${ }^{\circledR}$ ) peut être utilisé à la dose de $7 \mathrm{mg} / \mathrm{kg}$ (solution aqueuse à 7 p. 100) ; il procure une protection de trois semaines environ. Pendant la période d'hivernage, le chlorhydrate de chlorure d'isométamidium (Trypamidium ${ }^{\circledR}$ ) peut être utilisé à la dose de 0,5 à $1 \mathrm{mg} / \mathrm{kg}$ de poids vif pour couvrir toute la période de la campagne agricole ; il procure une protection de quatre mois en moyenne.

\section{Remerciements}

Les auteurs remercient l'Union européenne par l'intermédiaire du Réseau ouest-africain de la traction animale (Roata) qui a financé la première phase du projet, l'Ilri qui a financé la deuxième phase et tous ceux qui les ont aidés dans la réalisation de ce travail.

\section{BIBLIOGRAPHIE}

1. AGYEMANG K., DWINGER R.H., TOURAY B.N., JEANIN P., FOFANA D., GRIEVE A.S., 1980. Effects of nutrition on degree of anemia live weight changes in Ndama cattle infected with trypanosomes. Livest. Prod. Sci., 26: 39-51.

2. AOAC, 1990. Official methods of analysis. 15th edn. Gaithersburg, MD, USA, Association of Analytical Chemists.

3. CIPEA., 1990. Rapport annuel et synthèse des programmes. AddisAbéba, Ethiopie, CIPEA, 107 p.

4. CLAIR M., 1987. L'épidémiologie de la trypanosomose africaine. In : Production animale dans les régions d'Afrique infestées par les glossines. Addis-Abéba, Ethiopie, CIPEA/IIrad, p.85-95.

5. DIATTA A., 1984. Conditions de productions et d'exploitation des cultures fourragères d'Andropogon gayanus Kanth à Kolda (Casamane, République du Sénégal). Travail de fin d'études pour l'obtention de grade d'ingénieur agronome. Orientation : élevage. Gembloux, Belgique, faculté des Sciences agronomiques, $117 \mathrm{p}$

6. DRAWER K., 1959. Anspannung Und Beschirrung der Haustiere. Frankfurt, Deutschland, Verlag.

7. DWINGER R.H., CHIFFORD D., AGYEMANG K., GETTINBY G., GRIEVE A.S., KORA S., BOJANG M.A., 1992. Comparative study on Ndama and zebu cattle following repeated infection with Trypanosoma congolense. Res. vet. Sci., 52: 292-298.

8. FALL A., DIACK A., 1993. Productivité et pathologie du bétail Ndama. Site de Kolda. Entomologie et santé animale. Rapport Crz de Kolda. Kolda, Sénégal, Drpsa-Isra.

9. FAO., 1995. Animal production and health trypanomiasis ELISA Kit. Direct sandwich enzyme immunoassay for the detection of $T$. brucei, T. congolense and T. vivax. Rome, Italy, FAO, $37 \mathrm{p}$.

10. FAYE A., DIACK A., DIEYE P.N., FALL A., 1996. Projet de recherche sur les inter relations entre les pathologies parasitaires des bovins de trait en zone subhumide du Sénégal. Rapport d'avancement. Kolda, Sénégal, Centre de recherches zootechnique/lsra,10 $\mathrm{p}$.

11. HOPFEN H.J., 1969. Farm implements for arid and tropical regions. Rome, Italy, FAO. (Agricultural development paper No 91).

12. ILRAD, 1986. Rapport annuel. Naïrobi, Kenya, Laboratoire international pour la recherche sur les maladies animales, $94 \mathrm{p}$.

13. MURRAY M., MURRAY P.K., MCINTYRE W.I., 1977. An improved parasitological technique for the diagnostic of Africain trypanosomiasis. Trans. R. Soc. trop. Med. Hyg., 71: 325-326.

14. MURRAY M., TRAIL J.C.M., TURNER D.A., WISSOCQ Y., 1983. Productivité animale et trypanotolérance : manuel de formation pour les activités du Réseau. Addis-Abéba, Ethiopie, Centre international pour l'élevage en Afrique, 198 p.

15. NAYLOR D.C., 1971. The haematology and histopathology of Trypanosoma congolense infection in cattle. Part II. Haematology (including symptoms). Trop. Anim. Health Prod., 3: 159-168.

16. PARIS J., MURRAY M., MCODIMBA F., 1982. A comparative evolution of the parasitolagical techniques currently available for the diagnosis of African trypanosomiasis in cattle. Acta trop., 39: 307-316.

17. SAS/STAT, 1989. SAS/STAT User's Guide. Vers. 6, 4th edn, vol. 2 Cary, NC, USA, SAS Institute, 846 p.

Accepté le 26.06.2003 


\section{Summary}

Seck M.T., Fall A., Diaite A., Diokou A., Dieng M. Effect of Trypanosome Infection on Work Performance of Trypanotolerant Ndama Cattle in Senegal Subhumid Zone

The aim of this study was to assess trypanosome infection effects on the work performance of trypanotolerant cattle in the subhumid zone of Senegal. The study was conducted on Ndama cattle with a mean live weight of $288 \mathrm{~kg}$ and a mean age of six years. The animals were trained individually (single ox). The experimental design comprised two phases of four weeks each. In the first phase, the animals worked five hours a day, five days a week drafting the equivalent of $12 \%$ their body weight. A three-week rest period followed. Then, in the second phase, they were intradermally infected with a strain of Trypanosoma congolense (infective dose: $10^{5}$ trypanosomes per milliliter) and subjected to the same work. The trypanosome infection had a significant effect on work power $(P<0.001)$, work speed $(P<0.001)$, distance traveled $(P<0.001)$, volume of pellets obtained by centrifugation $(\mathrm{P}<0.05)$ and Andropogon gayanus intake $(p<0.01)$. However, the infection had no significant effect on the animals' live weights. Results showed the work performance of infection-free animals was better than that of trypanosome-infected cattle. Healthcare and prophylactic measures against trypanosomes are proposed to improve the work performance of trypanotolerant Ndama cattle used for drafting: cattle should be treated against trypanosomosis with diminazene aceturate (Bérénil $\left.{ }^{\circledR}\right)(7 \mathrm{mg} / \mathrm{kg}$ body weight) at the end of the dry season, and with isometamidium $(0,5-1 \mathrm{mg} / \mathrm{kg}$ body weight) during the rainy season, to protect them during the land preparation period.

Key words: N'Dama cattle - Disease resistance Trypanosoma congolense - Experimental infection - Animal performance - Senegal.

\section{Resumen}

Seck M.T., Fall A., Diaite A., Diokou A., Dieng M. Efecto de la infección por tripanosomas sobre los rendimientos de trabajo de los taurinos Ndama tripanotolerantes en la zona sub húmeda de Senegal

El presente estudio tuvo por objetivo el de medir el impacto de la infección por tripanosomas sobre los rendimientos en el trabajo de los taurinos tripanotolerantes en la zona sub húmeda de Senegal. Se llevó a cabo sobre machos Ndama con un peso vivo medio de $288 \mathrm{~kg}$ y una edad media de seis años. Los animales fueron criados en forma individual (mono buey). El dispositivo experimental se compuso de dos fases de cuatro semanas cada una. La primera consistió en utilizar animales sanos para tracción de una carga equivalente al $12 \%$ de su peso vivo durante cinco horas por día y cinco días por semana. Durante la segunda fase, después de tres semanas de reposo, los animales fueron infectados artificialmente por vía intradérmica con una cepa de Trypanosoma congolense (dosis infectante $10^{5}$ tripanosomas por mililitro) y fueron sometidos a los mismos ejercicios. La infección tripanosómica tuvo un efecto significativo sobre la potencia desarrollada $(p<0,001)$, la velocidad de trabajo $(p<0,001)$, la distancia recorrida $(p<0,001)$, el volumen del fondo de centrifugación $(\mathrm{p}<0,05)$ y el consumo de Andropogon gayanus $(\mathrm{P}<0,01)$. Por otro lado, no presentó efecto significativo sobre el peso vivo de los animales. Los resultados demuestran que los animales sanos tienen un mayor rendimiento al trabajo que los animales infectados por los tripanosomas. Se proponen recomendaciones concernientes a los cuidados sanitarios y de profilaxia tripanosómica con el fin de aumentar los rendimientos al trabajo de los taurinos Ndama tripanotolerantes de tiro: al final de la estación seca, tratar a los animales contra la tripanosomosis con aceturato de diminasen (Bérénil $\left.{ }^{\circledR}\right)$, con dosis de $7 \mathrm{mg} / \mathrm{kg}$ y durante el invierno tratarlos con isometamidium a dosis de 0,5 a $1 \mathrm{mg} / \mathrm{kg}$ de peso vivo para protegerlos durante el periodo de la campaña agrícola

Palabras clave: Ganado bovino N'Dama - Resistencía a la enfermedad - Trypanosoma congolense - Infección experimental - Desempeño animal - Senegal. 